\title{
Homogenization of degenerate second-order PDE in periodic and almost periodic environments and applications
}

\author{
Pierre-Louis Lions ${ }^{\mathrm{a}, \mathrm{b}, *}$, Panagiotis E. Souganidis ${ }^{\mathrm{c}, 1}$ \\ ${ }^{a}$ College de France, 11, place Marcelin Berthelot, Paris 75005, France \\ b CEREMADE, Université de Paris-Dauphine, place du Marechal de Lattre de Tassigny, Paris Cedex 16, France \\ ${ }^{\mathrm{c}}$ Department of Mathematics, The University of Texas at Austin, 1 University Station C1200, Austin, TX 78712-0257, USA
}

Received 25 October 2004; accepted 28 October 2004

Available online 1 August 2005

\begin{abstract}
We study the homogenization of fully nonlinear degenerate second-order pde, with "ellipticity" of the same order as the space oscillations, in periodic and almost periodic. As a special case we consider the class of quasi-linear, degenerate elliptic pde. The results apply to level sets equations describing the evolution of fronts with prescribed normal velocity. We also discuss an application about the averaged properties of interfacial motions in periodic and almost periodic environments.
\end{abstract}

(C) 2005 L'Association Publications de l'Institut Henri Poincaré. Published by Elsevier B.V. All rights reserved

\section{Introduction}

In this note we consider the behavior, as $\varepsilon \rightarrow 0$, of the solution $u^{\varepsilon} \in B U C\left(\mathbb{R}^{N}\right)$ of

$$
F\left(\varepsilon D^{2} u^{\varepsilon}, D u^{\varepsilon}, u^{\varepsilon}, x, \varepsilon^{-1} x\right)=0 \quad \text { in } \mathbb{R}^{N},
$$

where, if $S^{N}$ is the space of $N \times N$ symmetric matrices and $B U C(X)$ is the space of real valued bounded uniformly continuous functions defined on $X$ and \|\| is the sup-norm,

$$
\left\{\begin{array}{l}
F \in C_{\text {loc }}^{0,1}\left(S^{N} \times \mathbb{R}^{N} \times \mathbb{R} \times \mathbb{R}^{N} \times \mathbb{R}^{N}\right) \text { is degenerate elliptic, } \\
\text { i.e., for all } X, Y \in S^{N}, p, x, y \in \mathbb{R}^{N} \text { and } r \in \mathbb{R} \\
\text { if } X \leqslant Y, \quad \text { then } F(X, p, r, x, y) \geqslant F(Y, p, r, x, y)
\end{array}\right.
$$

\footnotetext{
* Corresponding author.

E-mail addresses: lions@ceremade.dauphine.fr (P.-L. Lions), souganid@ math.utexas.edu (P.E. Souganidis).

1 Partially supported by the National Science Foundation.
} 
The key property of $F$ which allows for the homogenization is a kind of "uniform" almost periodicity. The precise assumption is that

$$
\left\{\begin{array}{l}
\text { for all } x \in \mathbb{R}^{N}, r \in \mathbb{R} \text { and } R>0, \text { the family } \\
\left\{F(Q, p, x, r, \cdot+z): z \in \mathbb{R}^{N},(Q, p) \in \overline{B(0, R)} \times \overline{B(0, R)}\right\} \\
\text { is relatively compact in } B U C\left(\mathbb{R}^{N}\right) .
\end{array}\right.
$$

In other words, we assume that, for each fixed $x \in \mathbb{R}^{N}, r \in \mathbb{R}$ and $R>0$, given $\left(z_{j}\right)_{j \in \mathbb{N}} \subset \mathbb{R}^{N}$ such that, as $j \rightarrow \infty,\left|z_{j}\right| \rightarrow \infty$, the sequence $\left(F\left(Q, p, r, x, \cdot+z_{j}\right)\right)_{j \in \mathbb{N}}$ is relatively compact in $B U C\left(\mathbb{R}^{N}\right)$ uniformly in $(Q, p) \in \bar{B}(0, R) \times \bar{B}(0, R)$.

For $F$ independent of $Q, p, r$ and $x,(1.3)$ is one of the definitions for $F(Q, p, r, x, y)$ to be almost periodic with respect to the fast variable $y$. The additional requirement in (1.3) is that this property must hold, for each fixed $x$ and $r$, in a local uniform way with respect to the other variables. In the sequel, we call a function satisfying (1.3) almost periodic with respect to $y$ locally uniformly in $(Q, p)$.

The main result of the paper is that, under some additional technical assumptions on $F$, there exists $\bar{F}: \mathbb{R}^{N} \times \mathbb{R} \times$ $\mathbb{R}^{N} \rightarrow \mathbb{R}$ such that, as $\varepsilon \rightarrow 0, u^{\varepsilon} \rightarrow \bar{u}$ in $C\left(\mathbb{R}^{N}\right)$, where $\bar{u} \in B U C\left(\mathbb{R}^{N}\right)$ is the unique solution of the homogenized (averaged) pde

$$
\bar{F}(D \bar{u}, \bar{u}, x)=0 \quad \text { in } \mathbb{R}^{N} .
$$

Similar results hold for boundary value as well as time dependent versions of (1.1) with Dirichlet and nonoscillatory Neumann-type conditions. In particular, our results apply to the initial value problem

$$
\left\{\begin{array}{l}
u_{t}^{\varepsilon}+F\left(\varepsilon D^{2} u^{\varepsilon}, D u^{\varepsilon}, u^{\varepsilon}, x, \varepsilon^{-1} x, t\right)=0 \quad \text { in } \mathbb{R}^{N} \times(0, \infty), \\
u^{\varepsilon}=u_{0} \quad \text { on } \mathbb{R}^{N} \times\{0\},
\end{array}\right.
$$

and yield the effective equation

$$
\left\{\begin{array}{l}
\bar{u}_{t}+\bar{F}(D \bar{u}, \bar{u}, x, t)=0 \quad \text { in } \mathbb{R}^{N} \times(0, \infty), \\
\bar{u}=u_{0} \quad \text { on } \mathbb{R}^{N} \times\{0\} .
\end{array}\right.
$$

To simplify the presentation, we state results for the time independent problem (1.1). When we discuss the application to the interfacial motions, we will, however, use the evolution equation. Throughout the paper, to keep the ideas simple, we do not aim for the most general assumptions. Finally, we write $y$ to denote the "fast" variable $\varepsilon^{-1} x$.

To fix the ideas, we continue describing our results as they apply to general quasi-linear equations of the form

$$
-\varepsilon \operatorname{tr} A\left(D u^{\varepsilon}, x, \varepsilon^{-1} x\right) D^{2} u^{\varepsilon}+H\left(D u^{\varepsilon}, u^{\varepsilon}, x, \varepsilon^{-1} x\right)=0 \quad \text { in } \mathbb{R}^{N} \text {. }
$$

The assumptions on $H$ are:

$$
\begin{aligned}
& \left\{\begin{array}{l}
H \in C_{\text {loc }}^{0,1}\left(\mathbb{R}^{N} \times \mathbb{R}^{N} \times \mathbb{R}^{N} \times \mathbb{R}^{N}\right) \cap C^{0,1}\left(\overline{B(0, R)} \times \mathbb{R}^{N} \times \mathbb{R}^{N} \times \mathbb{R}^{N}\right), \\
\text { for each } R>0, \text { and, for each fixed } x \in \mathbb{R}^{N} \text { and } r \in \mathbb{R}, \\
H \text { is almost periodic with respect to } y,
\end{array}\right. \\
& \left\{\begin{array}{l}
\text { there exists } C>0 \text { such that, for all } p \in \mathbb{R}^{N}, r \in \mathbb{R} \text { and } x, \hat{x}, y, \hat{y} \in \mathbb{R}^{N}, \\
\left|D_{x} H\right|+\left|D_{y} H\right| \leqslant C(1+|H|),
\end{array}\right.
\end{aligned}
$$

and

$$
\left\{\begin{array}{l}
\text { there exists } \gamma>0 \text { such that, for all } p, x, y \in \mathbb{R}^{N} \text { and } r, s \in \mathbb{R} \text { with } r \geqslant s, \\
H(p, r, x, y)-H(p, s, x, y) \geqslant \gamma(r-s) .
\end{array}\right.
$$

As far as $A$ is concerned, we assume that

$$
\left\{\begin{array}{l}
A \in C^{0,1}\left(\mathbb{R}^{N} \times \mathbb{R}^{N} \times \mathbb{R}^{N} ; S^{N}\right) \text { and, for each } x \in \mathbb{R}^{N}, \\
A \text { is almost periodic with respect to } y \text { locally uniformly in } p
\end{array}\right.
$$


and, if $\mathcal{M}^{N \times M}$ is the space of $N \times M$ matrices,

$$
\left\{\begin{array}{l}
A=\Sigma \Sigma^{\mathrm{T}}, \text { where, for each } R>0, \\
\Sigma \in C_{\text {loc }}^{0,1}\left(\mathbb{R}^{N} \times \mathbb{R}^{N} \times \mathbb{R}^{N} ; \mathcal{M}^{N \times M}\right) \cap C^{0,1}\left(\overline{B(0, R)} \times \mathbb{R}^{N} \times \mathbb{R}^{N} ; \mathcal{M}^{N \times M}\right),
\end{array}\right.
$$

and

$$
\left\{\begin{array}{l}
\text { there exists } \theta \in(0,1) \text { such that } \\
\underline{\lim \mid \rightarrow \infty}|p|^{-2}\left(\theta(1-\theta) H^{2}+\theta\|\Sigma\|^{2} D_{y} H \cdot p\right)>\left\|D_{y} \Sigma\right\|^{2}\|\Sigma\|^{2}
\end{array}\right.
$$

where, given $\Sigma=\left(\sigma_{i j}\right) \in \mathcal{M}^{N \times M}$ and using the summation convention, $\|\Sigma\|=\sigma_{i j}^{2}$. Notice that, if $A \in S^{N}$ and $\Sigma \in \mathcal{M}^{N \times M}$ are such that $A=\Sigma \Sigma^{\mathrm{T}}$, then $A \geqslant 0$ and $\|\Sigma\|=\operatorname{tr} A$.

We remark that, if $H$ satisfies (1.9) and (1.13), it is immediate that, uniformly in $x, y$ and $r$,

$$
H(p, r, x, y) \rightarrow \infty, \quad \text { as }|p| \rightarrow \infty .
$$

If $A \in S^{N}$ satisfies (1.12), it is, of course, degenerate elliptic. Moreover, if $A \in C^{1,1}\left(\mathbb{R}^{N} ; S^{N}\right)$ is degenerate elliptic, a classical result (see, for example, Oleinik [32] and Freidlin [19]) yields that there exists $\Sigma \in C^{0,1}\left(\mathbb{R}^{N} ; S^{N}\right.$ ) such that

$$
A=\Sigma \Sigma^{\mathrm{T}} \quad \text { and, for all } X \in S^{N}, \quad\left|\operatorname{tr} D_{y} A X\right| \leqslant 2 N(\operatorname{tr} A X)^{1 / 2}\left\|D_{y}^{2} A\right\| .
$$

Finally, for future reference, we recall that, if $A, B \in S^{N}$ with $A \geqslant 0$ and $D, E \in \mathcal{M}^{N \times M}$, a simple application of Cauchy-Schwartz inequality yields that

$$
(\operatorname{tr} A B)^{2} \leqslant \operatorname{tr}(A B B) \operatorname{tr} A \quad \text { and } \quad\left(\operatorname{tr} D E^{\mathrm{T}} B\right)^{2} \leqslant \operatorname{tr}\left(D D^{\mathrm{T}} B B\right)\|E\|^{2} .
$$

Our result is:

Theorem 1.1. Assume (1.8)-(1.13). There exists $\bar{F} \in C_{\text {loc }}^{0,1}\left(\mathbb{R}^{N} \times \mathbb{R} \times \mathbb{R}^{N}\right)$ satisfying (1.9), (1.10) and (1.14) such that, if $u^{\varepsilon} \in B U C\left(\mathbb{R}^{N}\right)$ and $\bar{u} \in B U C\left(\mathbb{R}^{N}\right)$ solve respectively (1.7) and (1.4), then, as $\varepsilon \rightarrow 0$, $u^{\varepsilon} \rightarrow \bar{u}$ in $C\left(\mathbb{R}^{N}\right)$.

When $A$ is uniformly elliptic, Theorem 1.1 is a special case of [18]. When, however, $A$ is only degenerate elliptic, it is not possible to use the classical Krylov-Safonov-type estimates which provide uniform Holder-continuity. This is where (1.13) comes in. Indeed (1.13) is a technical hypothesis that we use in order to obtain appropriate uniform Lipschitz estimates in our proof. As we discuss at the end of Section 3, when $N=1,(1.13)$ is not necessary. We do not know, however, whether, when $N>1$, this condition is actually necessary for the homogenization of almost periodic quasi-linear equations. For further comments we refer to the discussion after Lemma 3.2.

The Lipschitz estimates we obtain using (1.13) are new in the context of quasilinear elliptic pde and generalize other estimates, which were obtained by Serrin [36] and one of the authors [25] under the assumption of uniform ellipticity.

Finally it is immediate that (1.13) holds for any $H$ satisfying (1.9) and grows superlinearly with respect to $p$, uniformly in $y$, i.e., uniformly in $y$ and as $|p| \rightarrow \infty$,

$$
|p|^{-1} H(p, y) \rightarrow \infty \text {. }
$$

In the statement of Theorem 1.1 we assume that there exists a solution $u^{\varepsilon} \in B U C\left(\mathbb{R}^{N}\right)$ of (1.7). To guarantee this fact without restricting the growth of $H$, we need to change (1.13) to include some information about the dependence of $A$ and $H$ on $x$. In particular, to obtain, for each fixed $\varepsilon>0$, Lipschitz continuous solutions of (1.7), we assume that

$$
\left\{\begin{array}{l}
\text { for each } \varepsilon>0, \text { there exists } \theta_{\varepsilon} \in(0,1) \text { such that } \\
\underline{\lim _{|p| \rightarrow \infty}}|p|^{-2}\left(\theta_{\varepsilon}\left(1-\theta_{\varepsilon}\right) H^{2}+\theta_{\varepsilon}\|\Sigma\|^{2}\left(D_{y} H+\varepsilon D_{x} H\right) \cdot p\right)>\left\|\left(D_{y}+\varepsilon D_{x}\right) \Sigma\right\|^{2}\|\Sigma\|^{2} .
\end{array}\right.
$$


A variant of Theorem 1.1 applies to geometric equations, a special class of quasi-linear evolution equations describing the propagation of interfaces with prescribed normal velocity. Geometric equations do not satisfy all the assumptions of the above theorem, since, in this case, $A$ is discontinuous when $p=0$. A straightforward approximation argument together with the stability properties of the viscosity solutions takes care of this difficulty.

Although our results apply to more general geometric equations, to keep the presentation simple, here we consider the particular example

$$
\left\{\begin{array}{l}
u_{t}^{\varepsilon}-\varepsilon \delta \operatorname{tr}\left[\left(I-\widehat{D u^{\varepsilon}} \otimes \widehat{D u^{\varepsilon}}\right) D^{2} u^{\varepsilon}\right]+a\left(\varepsilon^{-1} x\right) H\left(D u^{\varepsilon}\right)=0 \quad \text { in } \mathbb{R}^{N} \times(0, \infty), \\
u^{\varepsilon}=u_{0} \quad \text { on } \mathbb{R}^{N} \times\{0\}
\end{array}\right.
$$

where, for $p \in \mathbb{R}^{N} \backslash\{0\}, \hat{p}=|p|^{-1} p, \delta>0$ and

$$
\begin{aligned}
& \left\{\begin{array}{l}
H \in C^{0,1}\left(\mathbb{R}^{N} ;[0, \infty)\right) \text { is positively homogeneous of degree } 1 \\
\text { with respect to } p, \text { i.e., there exists } v \in C^{0,1}(\partial B(0,1) ;(0, \infty)) \\
\text { such that, for all } p \in \mathbb{R}^{N} \backslash\{0\}, H=|p| v(\hat{p}),
\end{array}\right. \\
& a \in C^{0,1}\left(\mathbb{R}^{N}\right) \text { is almost periodic and } \inf a \geqslant 0,
\end{aligned}
$$

and

$$
\inf \left(a^{2} v-(N-1) \delta\left|D_{y} a\right|\right)>0 .
$$

It turns out (see [37,38] for a general discussion) that the set $\Gamma_{+}^{\varepsilon}=\left\{x \in \mathbb{R}^{N}: u^{\varepsilon}(x, t)=0\right\}$ is the generalized evolution of $\Gamma_{0}^{\varepsilon}=\left\{x \in \mathbb{R}^{N}: u_{0}(x)=0\right\}$ with normal velocity

$$
V=-\varepsilon \delta \operatorname{tr} D n-a\left(\varepsilon^{-1} x\right) H(-D n) .
$$

The result is:

Proposition 1.2. Assume (1.18), (1.19) and (1.20). For each $\delta>0$, there exists $\bar{H}_{\delta} \in C^{0,1}\left(\mathbb{R}^{N} ;[0, \infty)\right)$ satisfying (1.18) such that, if $u^{\varepsilon}$ and $\bar{u}$ solve (1.17) and (1.6) with $\bar{F}=\bar{H}_{\delta}$ and initial datum $u_{0}^{\varepsilon}$ and $u_{0}$ in BUC $\left(\mathbb{R}^{N}\right)$ respectively, then, if, as $\varepsilon \rightarrow 0, u_{0}{ }^{\varepsilon} \rightarrow u_{0}$ in $C\left(\mathbb{R}^{N}\right)$, then $u^{\varepsilon} \rightarrow \bar{u}$ in $C\left(\mathbb{R}^{N} \times[0, \infty)\right)$. In particular, if $\left(\Gamma_{t}^{\varepsilon}\right)_{t} \geqslant 0$ and $\left(\bar{\Gamma}_{\underline{t}}\right)_{t} \geqslant 0$ are the generalized evolutions of $\Gamma_{0}=\left\{x \in \mathbb{R}^{N}: u_{0}(x)=0\right\}$ with normal velocities given by (1.21) and $-\bar{H}_{\delta}(-D n)$ respectively, then, as $\varepsilon \rightarrow 0, \Gamma_{t}^{\varepsilon} \rightarrow \bar{\Gamma}_{t}$ in the Hausdorff metric.

Assumption (1.20) corresponds, in the setting of geometric equations, to (1.13). We do not know whether (1.20) is necessary for the above result or whether inf $a>0$ is enough, as it is the case when there is no second-order term in (1.17) (we refer to $[5,16,29,30]$ for a detailed discussion of the case of only first-order velocity). Note that when $N=1$ or $\delta=0,(1.20)$ reduces to the usual assumption that inf $a>0$. If (1.20) is indeed necessary, this may be related to issues related to "pinning" of interfaces [15,31].

We continue with the result for the general problem (1.1). We need to assume that

$$
\begin{aligned}
& \left\{\begin{array}{l}
\text { there exists } \rho \in C([0, \infty) ;[0, \infty)) \text { with } \rho(0+) \text { such that } \\
F(X, \alpha(x-y), r, x, y, \omega)-F(Y, \alpha(x-y), r, \hat{x}, \hat{y}, \omega) \\
0 \leqslant \rho\left(\alpha|x-\hat{x}|^{2}+|x-\hat{x}|+\beta|y-\hat{y}|^{2}+|y-\hat{y}|\right) \\
\text { for all } \alpha, \beta>0, r \in \mathbb{R}, x, y, \hat{x}, \hat{y} \in \mathbb{R}^{N} \text { and } X, Y \in S^{N} \text { such that } \\
-3(\alpha+\beta)\left(\begin{array}{cc}
I & 0 \\
0 & I
\end{array}\right) \leqslant\left(\begin{array}{cc}
X & 0 \\
0 & -Y
\end{array}\right) \leqslant 3(\alpha+\beta)\left(\begin{array}{cc}
I & -I \\
-I & I
\end{array}\right),
\end{array}\right. \\
& \left\{\begin{array}{l}
\text { there exists a constant } \gamma>0 \text { such that, for all } \\
(X, p, x, y) \in S^{N} \times \mathbb{R}^{N} \times \mathbb{R}^{N} \times \mathbb{R}^{N} \text { and } r, s \in \mathbb{R} \text { with } r \geqslant s, \\
F(X, p, r, x, y)-F(X, p, s, x, y) \geqslant \gamma(r-s)
\end{array}\right.
\end{aligned}
$$


and

$$
\left\{\begin{array}{l}
\text { there exist positive constants } L \text { and } R \text { such that, for all } \\
(X, p, r, x, y) \in S^{N} \times \mathbb{R}^{N} \times \mathbb{R} \times \mathbb{R}^{N} \times \mathbb{R}^{N} \text { with }|p|>R, \\
\text { if }-\operatorname{tr}\left(D_{X} F(X, p, r, x, y) X X\right)+D_{y} F(X, p, r, x, y) \cdot p \leqslant 0 \\
\text { and } F(X, p, r, x, y) \leqslant R, \quad \text { then }|p| \leqslant L .
\end{array}\right.
$$

Hypotheses (1.2), (1.22) and (1.23) are the standard assumptions (see [13]) which guarantee, for each $\varepsilon>0$, the existence and uniqueness of viscosity solutions of (1.1). Assumption (1.24) is used to obtain Lipschitz bounds on the solutions of (1.1) which are independent of $\varepsilon$. In the case of the quasi-linear problem (1.7), the assertion of (1.24) follows from (1.12) and (1.13) and, in some sense, incorporates the conclusions one obtains using (1.24) in the quasi-linear setting.

To justify the Lipschitz bounds it is necessary to use either the classical Bernstein method, which, however, requires that the solutions and $F$ are at least $C^{2}$, or some more involved arguments from the theory of viscosity solutions (weak Bernstein method), like the ones introduced by Ishii and Lions [21] and Barles [3] to study similar questions. The $C^{2}$-regularity can be achieved if $F$ is either convex or concave and uniformly elliptic with respect to $X$, while, in the general case, it is not known. Hence for non convex $F$, it is necessary to follow [3,21], etc., or to come up with some approximations which have the needed properties. In this paper, to keep the presentation simple, we use the classical Bernstein method assuming tacitly that we have the necessary regularity. Of course in the case of quasi-linear equations, the regularity can be achieved by straightforward regularizations.

Our main result is:

Theorem 1.3. Assume (1.2), (1.3), (1.22), (1.23) and (1.24). There exists $\bar{F} \in C_{\mathrm{loc}}^{0,1}\left(\mathbb{R}^{N} \times \mathbb{R} \times \mathbb{R}^{N}\right)$ satisfying (1.22) and (1.23) such that, if $u^{\varepsilon}, \bar{u} \in B U C\left(\mathbb{R}^{N}\right)$ solve (1.1) and (1.4) respectively, then, as $\varepsilon \rightarrow 0, u^{\varepsilon} \rightarrow \bar{u}$ in $C\left(\mathbb{R}^{N}\right)$.

The paper is organized as follows: Section 2 is devoted to a brief review and a discussion of recent advances of the theory of homogenization of fully nonlinear first- and second-order pde in periodic, almost periodic and stationary ergodic environments. We also discuss why the previously known theory does imply the results we present here. Section 3 is devoted to the main estimate leading to Theorem 1.3 as well as a sketch of the proofs of Theorem 1.1 and Proposition 1.2.

\section{A review and recent advances in the theory of homogenization}

There is an extensive literature about the homogenization of nonlinear first- and second-order pde in periodic and almost periodic settings. Lions et al. [29] were the first to consider the homogenization of Hamilton-Jacobi equations. This problem was revisited by Evans [17,18], who introduced the method of the perturbed test function for viscosity solutions and considered a number of examples for second-order pde including (1.1), but under the assumption that $F$ is uniformly elliptic. We also refer to Caffarelli [8], Majda and Souganidis [30], and, more recently, Arisawa [1], Arisawa and Lions [2], Cocordel [12], E [16] and Bhattacharya and Cracium [5] for other results always in the periodic setting. Ishii in [20] considered the homogenization of Hamilton-Jacobi equations in almost periodic settings. There are not, however, many results for the homogenization of degenerate second-order pde in either setting except of the work of Arisawa and Lions [2], which, however, does not cover the results of the paper at hand.

Recently, there has been a resurgence of interest in the homogenization of first- and second-order pde in random stationary ergodic media. This is a very general setting lacking the compactness properties used extensively in various places in the study of the periodic/almost periodic homogenization. To overcome this difficulty, it is necessary to employ the ergodic theorem in various places. The literature in the random setting is far more limited. Papanicolaou and Varadhan [33,34] and Kozlov [24] (see also Jikov et al. [22]) were the first to consider the homogenization of linear uniformly elliptic operators. Their results were extended to particular quasi-linear problems 
by Bensoussan and Blakenship [4] and Castel [11]. We also refer to Bourgeat and Piatniski [6] for some more recent results in the linear case as well as more references. The first nonlinear result in a variational setting was obtained by Dal Maso and Modica [14].

The homogenization of fully nonlinear, convex, first-order (Hamilton-Jacobi) equations in random environments was considered first by one of the authors [39] (see also [35]). In a subsequent work [27], the authors showed that in this case, in general, there are no correctors, i.e., solutions, of the associated macroscopic problem. The homogenization of fully nonlinear, uniformly elliptic equations was studied by Caffarelli, Souganidis and Wang in [10]. In a forthcoming paper [9], Caffarelli and the authors show that correctors exist for convex, fully nonlinear, uniformly elliptic equations. Recently the authors studied, in [26], always in the stationary ergodic setting, the homogenization of (1.7), when the matrix $A$ is independent of $p$ and $H$ is convex. Both assumptions are necessary for the techniques of [26], which uses the sub-additive ergodic theorem, the stochastic control formulation of the problem, hence the assumptions of the convexity of $H$ and the independence of $A$ on $p$, together with a number of new Lipschitz estimates similar to the one's in this paper, which do not depend on these restrictions. More recently, Kosynga et al. [23] considered, using different methods, a problem similar to the one of [26] under the stronger assumptions of uniform ellipticity on $A$ and strict convexity of $H$. We are not aware, however, of any results for (1.1) in the random setting even for uniformly elliptic $F$.

Going back to the periodic/almost periodic setting we remark that the case at hand, i.e., degenerate elliptic equations, like (1.1), (1.7) and (1.17), was not covered by any previous work and in any setting. The closest results are the ones of Section 8 of [18], which, however, assume that $F$ is uniformly elliptic and, hence, exclude the important case of geometric equations. The techniques of [18] rely heavily on this assumption and, hence, cannot be relaxed without new estimates. Although it is possible to obtain in some cases bounds for approximate equations, the estimates depend strongly on and degenerate at the limit of the approximations.

\section{Sketch of the proofs}

We begin with Theorem 1.3 and concentrate first on the periodic setting. Using appropriate (perturbed) test functions (see $[29,17,18,30]$, etc.), it follows that it suffices to solve the associated cell problem, i.e., to find for each $(p, r, x) \in \mathbb{R}^{N} \times \mathbb{R} \times \mathbb{R}^{N}$, a unique constant $\bar{F}(p, r, x)$ such that the equation

$$
F\left(D^{2} v, D v+p, r, x, y\right)=\bar{F}(p, r, x) \quad \text { in } \mathbb{R}^{N}
$$

admits a solution $v \in B U C\left(\mathbb{R}^{N}\right)$. This is the object of the next result.

Proposition 3.1. Assume (1.2), (1.3) (with F periodic), (1.22), (1.23) and (1.24). For each $(p, r, x) \in \mathbb{R}^{N} \times \mathbb{R} \times \mathbb{R}^{N}$, there exists a unique constant $\bar{F}(p, r, x)$ such that (3.1) has a solution $v \in B U C\left(\mathbb{R}^{N}\right)$.

The uniqueness of the constant is a consequence of the theory of viscosity solution (see, for example, [13]), once it is established that there exists a solution $v \in B U C\left(\mathbb{R}^{N}\right)$ of (3.1). Note that, since $F$ is periodic, (3.1) is actually solved only in the periodic domain, hence the name cell problem. In the almost periodic as well as general stationary ergodic settings, (3.1) has to be considered in the whole space. We believe that in such settings it is more appropriate to call (3.1) the macroscopic problem. In this case, to guarantee the uniqueness of $\bar{F}(p, r, x)$, it suffices to find solutions $v \in U C\left(\mathbb{R}^{N}\right)$ with strictly sub-linear growth at infinity—see [27,26] and [39]. Below we concentrate on the issue of the existence of solutions $v \in B U C\left(\mathbb{R}^{N}\right)$ in the periodic setting.

To simplify the presentation, in what follows, we assume that

$$
F \in C^{2}\left(S^{N} \times \mathbb{R}^{N} \times \mathbb{R} \times \mathbb{R}^{N} \times \mathbb{R}^{N}\right) \text { and } v_{\lambda} \in C^{2}\left(\mathbb{R}^{N}\right) .
$$

Otherwise we either approximate and regularize the equation and obtain a priori bounds independent of the regularization or alternatively we use the weak Bernstein method. (See the discussion before the statement of Theorem 1.3.) Moreover, since $p, r$ and $x$ are fixed, we drop next the explicit dependence on them. 
We begin with the approximate problem

$$
\lambda v_{\lambda}+F\left(D^{2} v_{\lambda}, D v_{\lambda}, y\right)=0 \quad \text { in } \mathbb{R}^{N},
$$

which, in view of the theory of viscosity solutions (see [13]), admits a unique periodic continuous solution $v_{\lambda} \in$ $B U C\left(\mathbb{R}^{N}\right)$ satisfying the bound

$$
\sup _{\lambda>0}\left\|\lambda v_{\lambda}\right\|<\infty \text {. }
$$

It is worth remarking that (3.3) is an approximation which is closely related to homogenization. Indeed, if $v^{\lambda}=\lambda v_{\lambda}\left(\lambda^{-1} y\right)$, then

$$
v^{\lambda}+F\left(\lambda D^{2} v^{\lambda}, D v^{\lambda}, \lambda^{-1} y\right)=0
$$

and the limiting behavior of the $v^{\lambda}$ s, as $\lambda \rightarrow 0$, is equivalent to the limiting behavior, again as $\lambda \rightarrow 0$, of the $\lambda v^{\lambda}$ s in balls of radius $\mathrm{O}\left(\lambda^{-1}\right)$.

Consider next the function $\hat{v}_{\lambda}=v_{\lambda}-v_{\lambda}(0)$, which solves the equation

$$
\lambda \hat{v}_{\lambda}+F\left(D^{2} \hat{v}_{\lambda}, D \hat{v}_{\lambda}, y\right)=-\lambda v_{\lambda}(0) \quad \text { in } \mathbb{R}^{N} .
$$

Since $\lambda v_{\lambda}(0)$ is bounded, the claim will follow, if we show that along subsequences $\lambda_{n} \rightarrow 0, \hat{v}_{\lambda_{n}} \rightarrow \hat{v}$ in $C\left(\mathbb{R}^{N}\right)$ for some $\hat{v} \in B U C\left(\mathbb{R}^{N}\right)$. This in turn follows, if it is shown that the family $\left(v_{\lambda}\right)_{\lambda>0}$ is equi-continuous. Notice that the equi-continuity of the $v_{\lambda}$ 's also establishes, in view of the assumption of periodicity, the bound on the $\hat{v}_{\lambda}$ 's and $\hat{v}$. In the uniformly elliptic case this is, of course, provided by the regularity theory of such equations (see Cabre and Caffarelli [7]), and the bound depends on the ellipticity constants. This is the reason that the result of this note does not follow by regularizing (1.1) to a uniformly elliptic equation and using the stability properties of the viscosity solutions. The bound deteriorates as the ellipticity constant goes to zero.

It is therefore necessary to obtain a modulus of continuity directly for the degenerate case. This is the object of the next lemma, which yields a uniform Lipschitz bound.

Lemma 3.2. Assume (1.2), (1.22) and (1.24). There exists a constant $L>0$ such that, for all $x, y \in \mathbb{R}^{N}$,

$$
\left|v_{\lambda}(x)-v_{\lambda}(y)\right| \leqslant L|x-y| \text {. }
$$

Note that we do not assume (1.3), since the periodic/almost periodic structure of $F$ plays absolutely no role in the proof. Before we present the proof of the lemma, we remark that, of course, the $v_{\lambda}$ 's can be uniformly equicontinuous without being uniformly Lipschitz continuous. We refer to [28] for a number of explicit examples. As discussed in the Introduction we do not know whether (1.24) is necessary for the homogenization. It is shown, however, in [28] that (1.24) is necessary and sufficient for the above lemma to hold.

Proof of Lemma 3.2. 1. To simplify the presentation below we write $v$ instead of $v_{\lambda}$ and assume that we have the regularity asserted in (3.2).

2. Let $w=|D v|^{2}$. A straightforward differentiation yields,

$$
2 \lambda w+\operatorname{tr}\left(D_{X} F D^{2} w\right)-2\left(\operatorname{tr} D_{X} F D^{2} v D^{2} v\right)+2 D_{y} F \cdot D v+D_{p} F \cdot D w=0,
$$

and, hence, at a maximum point of $w$,

$$
\lambda w-\operatorname{tr} D_{X} F D^{2} v D^{2} v+D_{y} F \cdot D v \leqslant 0 .
$$

Moreover (3.3) and (3.4) yield that $F \leqslant R=\left\|\lambda v_{\lambda}\right\|$.

3. It follows from (1.24), that there exists a constant $L$ such that the claim holds. 
Next we discuss the extension to the almost periodic setting. It turns out that, in this case, it suffices to find "approximate correctors", a fact which is equivalent to showing that, for each $(p, r, x) \in \mathbb{R}^{N} \times \mathbb{R} \times \mathbb{R}^{N}$, as $\lambda \rightarrow 0$, $\lambda v_{\lambda} \rightarrow-\bar{H}(p, r, x)$ or $\lambda \hat{v}_{\lambda} \rightarrow 0$ uniformly on $\mathbb{R}^{N}$.

In our context this can be shown by a straightforward modification of the arguments of [20]. We have

Lemma 3.3. Assume (1.2), (1.3), (1.22) and (1.24) and let $\hat{v}_{\lambda}$ be the solution of (3.3). Then, as $\lambda \rightarrow 0, \lambda \hat{v}_{\lambda} \rightarrow 0$, uniformly in $\mathbb{R}^{N}$.

Proof. 1 . We argue by contradiction and we assume, for definiteness, that there exist $\theta>0, \lambda_{j} \rightarrow 0$ and $y_{j} \in \mathbb{R}^{N}$ such that, as $j \rightarrow \infty$,

$$
\lambda_{j} \hat{v}_{\lambda_{j}}\left(y_{j}\right) \geqslant 4 \theta \text { and }\left|y_{j}\right| \rightarrow \infty \text {. }
$$

2. The functions $\tilde{v}_{j}(y)=\hat{v}_{\lambda_{j}}\left(y+y_{j}-y_{k}\right)$ satisfy the equation

$$
0=\lambda_{j} \tilde{v}_{j}+F\left(D^{2} \tilde{v}_{j}, p+D \tilde{v}_{j}, y+y_{j}-y_{k}\right)+\lambda_{j} v_{j}(0) .
$$

Moreover, Lemma 3.2 yields a, uniform in $\lambda$, Lipschitz bound with constant $L$ for the $\hat{v}_{\lambda}$ 's and, hence, $\tilde{v}_{j}$ 's.

3. The proof of the comparison principle of viscosity solutions (see [13]) yields, for each $\alpha>0$, the estimate

$$
\lambda_{j} \tilde{v}_{j} \leqslant \lambda_{j} \hat{v}_{\lambda_{j}}+\rho\left(L \alpha^{-1}\right)+L_{F, k, j}^{\alpha, L} \text { in } \mathbb{R}^{N},
$$

where $\rho$ is as in (1.22) and

$$
L_{F, k, j}^{\alpha, L}=\sup \left\{\left|F\left(Q, p, y+y_{k}\right)-F\left(Q, p, y+y_{j}\right)\right|:\|Q\| \leqslant 3 \alpha,|p| \leqslant L, y \in \mathbb{R}^{N}\right\} .
$$

Choose next $\alpha$ sufficiently large so that

$$
\rho\left(L \alpha^{-1}\right) \leqslant \theta
$$

and, since $F$ satisfies (1.3) with $R=\max (3 \alpha, L)>0$, choose sufficiently large $k$ and $j$ so that

$$
L_{F, k, j}^{\alpha, L} \leqslant \theta .
$$

It follows that

$$
\lambda_{j} \tilde{v}_{j} \leqslant \lambda_{j} \hat{v}_{\lambda_{j}}+2 \theta \text { in } \mathbb{R}^{N} .
$$

4. Evaluating the above inequality at $y=y_{k}$ and using the uniform in $\lambda$ Lipschitz continuity of $\hat{v}_{\lambda}$ with constant $L$ lead to

$$
\lambda_{j} \hat{v}_{\lambda_{j}}\left(y_{j}\right) \leqslant \lambda_{j} \hat{v}_{\lambda_{j}}\left(y_{k}\right)+\theta \leqslant \lambda_{j} L\left|y_{k}\right|+2 \theta,
$$

which gives a contradiction as $\lambda_{j} \rightarrow 0$.

We continue with the

Proof of Theorem 1.1. 1. It suffices to show that

$$
F(X, p, r, x, y)=-\operatorname{tr} A(p, x, y) X+H(p, r, x, y)
$$

satisfies the assumptions of Theorem 1.1. Indeed (1.2), (1.3), (1.22) and (1.23) are immediate. In the case at hand, the quantity in (1.24) is

$$
-\operatorname{tr}\left(D_{X} F X X\right)+D_{y} F \cdot p=\operatorname{tr}(A X X)-\operatorname{tr}\left(D_{y} A X\right) \cdot p+D_{y} H \cdot p .
$$

3. If

$$
-\operatorname{tr}\left(D_{X} F X X\right)+D_{y} F \cdot p \leqslant 0,
$$


then

$$
\operatorname{tr} A X X+D_{y} H \cdot p \leqslant \operatorname{tr}\left(D_{y} A X\right) \cdot p=\operatorname{tr}\left(\left(D_{y} \Sigma\right) \Sigma^{\mathrm{T}} X\right) \cdot p+\operatorname{tr}\left(\Sigma\left(D_{y} \Sigma^{\mathrm{T}}\right) X\right) \cdot p=2 \operatorname{tr}\left(\left(D_{y} \Sigma\right) \Sigma^{\mathrm{T}} X\right) \cdot p,
$$

and, in view of (1.16),

$$
\operatorname{tr}(A X X)+D_{y} H \cdot p \leqslant 2\left\|D_{y} \Sigma\right\|\left(\operatorname{tr} \Sigma \Sigma^{\mathrm{T}} X X\right)^{1 / 2}|p|=2\left\|D_{y} \Sigma\right\|(\operatorname{tr} A X X)^{1 / 2}|p| .
$$

Hence, for all $\theta \in(0,1)$,

$$
\operatorname{tr}(A X X)+D_{y} H \cdot p \leqslant \theta \operatorname{tr}(A X X)+\left\|D_{y} \Sigma\right\|^{2}|p|^{2} .
$$

If $F \leqslant R$, then

$$
H-R \leqslant \operatorname{tr}(A X),
$$

and, in view of (1.16),

$$
(H-R)_{+} \leqslant(\operatorname{tr} A X X)^{1 / 2}\|\Sigma\|,
$$

Hence, for all $\theta \in(0,1)$,

$$
\theta(1-\theta)\|\Sigma\|^{-2}(H-R)_{+}^{2}+\theta D_{y} H \cdot p \leqslant \theta^{-1}\left\|D_{y} \Sigma\right\|^{2}|p|^{2} .
$$

Finally using in above estimate the particular $\theta$ for which (1.13) holds, we see that there exists some $L>0$ such that

$$
|D v| \leqslant L
$$

The growth condition (1.13) is, of course, sufficient for the arguments we are using. It is, however, an interesting question to see whether they are, in general, actually necessary for the homogenization to hold, especially since in the case of the front propagation it yields (1.19).

We remark, for example, that for $N=1,(1.13)$ is not necessary. Indeed consider the equation

$$
-a\left(y, v^{\prime}\right) v^{\prime \prime}+b(y)\left|v^{\prime}\right|=0 \quad \text { in } \mathbb{R}
$$

with $b>0$. It is immediate that $\inf b>0$ yields a Lipschitz bound on $v$. It is shown, however, in [28] that (1.13) is indeed necessary to have uniform Lipschitz estimates for $N>1$.

We continue with the

Proof of Proposition 1.2. 1. We refer to $[38,39]$ for a discussion about how the homogenization of the level set equations yields the asserted convergence of the interfaces.

2. For the homogenization it suffices to check that (1.12) and (1.13) hold. This follows from a straightforward computation.

Indeed in this setting, for $p \in \mathbb{R}^{N} \backslash\{0\}$, we have

$$
\left\{\begin{array}{l}
A(p)=\delta(I-\hat{p} \otimes \hat{p}), \quad \Sigma(p)=\delta^{1 / 2}(I-\hat{p} \otimes \hat{p}), \quad \text { and } \\
H(p, y)=a(y)|p| v(\hat{p}), \quad D_{y} H=D_{y} a|p| v(\hat{p}) \quad \text { and } \quad\|\Sigma\|^{2}=\delta(N-1) .
\end{array}\right.
$$

In what follows, to simplify the presentation, we argue as if $A, \sigma, a$ and $v$ were smooth.

3. With the above particular form of $A$, (3.8) reads

$$
\delta \operatorname{tr}(A X X)+D_{y} a \cdot p|p| v \leqslant 0
$$

Repeating the argument of the proof of Theorem 1.1, we find that there exists $R>0$ such that, for all $\theta \in(0,1)$,

$$
(a v|p|-R)_{+}^{2}+\|\Sigma\|^{2} D_{y} a \cdot p|p| v \leqslant 0 .
$$

Then (1.20) yields the desired bound. 


\section{References}

[1] M. Arisawa, Quasi-periodic homogenizations for second-order Hamilton-Jacobi-Bellman equations, Adv. Math. Sci. Appl. 11 (2001) 465-480.

[2] M. Arisawa, P.-L. Lions, On ergodic stochastic control, Comm. Partial Differential Equations 23 (1998) 2187-2217.

[3] G. Barles, A weak Bernstein method for fully nonlinear elliptic equations, Differential Integral Equations 4 (1991) $241-262$.

[4] A. Bensoussan, G. Blakenship, Controlled diffusions in a random medium, Stochastics 24 (1988) 87-120.

[5] K. Bhattacharya, B. Cracium, Homogenization of a Hamilton-Jacobi equation associated with the geometric motion of an interface, Proc. Roy. Soc. Edinburgh Sect. A 133 (2003) 773-805.

[6] A. Bourgeat, A. Piatniski, Approximations of effective coefficients in stochastic homogenization, Ann. Inst. H. Poincaré Probab. Statist. 40 (2004) $153-165$.

[7] X. Cabre, L.A. Caffarelli, Fully nonlinear elliptic partial differential equations, Amer. Math. Soc., 1997.

[8] L.A. Caffarelli, A note on nonlinear homogenization, Comm. Pure Appl. Math. 52 (1999) 829-838.

[9] L.A. Caffarelli, P.-L., Lions, P.E. Souganidis, in preparation.

[10] L.A. Caffarelli, P.E. Souganidis, L. Wang, Stochastic homogenization for fully nonlinear, second-order partial differential equations, Comm. Pure Appl. Math. LVII (2005) 319-361.

[11] F. Castell, Homogenization of random semilinear PDEs, Probab. Theory Related Fields 121 (2001) 492-524.

[12] M. Cocordel, Periodic homogenization of Hamilton-Jacobi equations: additive eigenvalue and variational formulas, Indiana Univ. Math. J. 45 (1996) 1095-1117.

[13] M.G. Crandall, H. Ishii, P.-L. Lions, User's guide to viscosity solutions of second order partial differential equations, Bull. Amer. Math. Soc. 27 (1992) 1-67.

[14] G. Dal Maso, L. Modica, Nonlinear stochastic homogenization and ergodic theory, J. Reine Angew. Math. 368 (1986) $28-42$.

[15] N. Dirr, A. Yip, personal communication.

[16] W. E, A class of homogenization problems in the calculus of variations, Comm. Pure Appl. Math. XLIV (1991) 733-759.

[17] L.C. Evans, Periodic homogenization of certain fully nonlinear partial differential equations, Proc. Roy. Soc. Edinburgh Sect. A 120 (1992) $245-265$.

[18] L.C. Evans, The perturbed test function method for viscosity solutions of nonlinear pde, Proc. Roy. Soc. Edinburgh Sect. A 111 (1989) $359-375$

[19] M. Freidlin, On factorization of a non-negative definite matrix, Probab. Theory Appl. 13 (1968) 375-378 (in Russian).

[20] H. Ishii, Almost periodic homogenization of Hamilton-Jacobi equations, in: Int. Conf. on Diff. Eqs., vol. 1, Berlin 1999, World Scientific, River Edge, NJ, 2000, pp. 600-605.

[21] H. Ishii, P.-L. Lions, Viscosity solutions of fully nonlinear second-order elliptic partial differential equations, JDE 83 (1990) 26-78.

[22] V.V. Jikov, S.M. Kozlov, O.A. Oleinik, Homogenization of Differential Operators and Integral Functionals, Springer-Verlag, 1991.

[23] H. Kosynga, F. Rezankhanlou, S.R.S. Varadhan, Stochastic homogenization of Hamilton-Jacobi-Bellman equations, Preprint.

[24] S.M. Kozlov, The method of averaging and walks in inhomogeneous environments, Russian Math. Surveys 40 (1985) $73-145$.

[25] P.-L. Lions, Resolution de problemes elliptic quasilineaires, Arch. Rational Mech. Anal. 74 (1980) 335-353.

[26] P.-L. Lions, P.E. Souganidis, Correctors for the homogenization of Hamilton-Jacobi equations in a stationary ergodic setting, Comm. Pure Appl. Math. LVI (2003) 1501-1524.

[27] P.-L. Lions, P.E. Souganidis, Homogenization of “viscous" Hamilton-Jacobi equations in stationary ergodic media, Comm. Partial Differential Equations 30 (2005) 335-375.

[28] P.-L. Lions, P.E. Souganidis, in preparation.

[29] P.-L. Lions, G. Papanicolaou, S.R.S. Varadhan, Homogenization of Hamilton-Jacobi equations, Preprint.

[30] A. Majda, P.E. Souganidis, Large scale front dynamics for turbulent reaction-diffusion equations with separated velocity scales, Nonlinearity 7 (1994) $1-30$.

[31] S. Müller, private communication.

[32] A. Oleinik, Alcuni visultati sulle equazioni lineari e quasilineri ellitico-paraboliche a derivate parziali del second ordine, Rend. Classe Sci. Fis. Mat., Nat. Acad. Naz. Lincei, Sci. 840 (1966) 774-784.

[33] G. Papanicolaou, S.R.S. Varadhan, Boundary value problems with rapidly oscillating random coefficients, in: J. Fritz, J.L. Lebaritz, D. Szasz (Eds.), Rigorous Results in Statistical Mechanics and Quantum Field Theory, in: Proc. Colloq. on Random Fields, Colloquia Mathematica Societ. Janos Bolyai, vol. 10, 1979, pp. 835-873.

[34] G. Papanicolaou, S.R.S. Varadhan, Diffusion with random coefficients, in: P.R. Krishnaiah (Ed.), Essays in Statistics and Probability, North-Holland, 1981.

[35] F. Rezankhanlou, J. Tarver, Homogenization for stochastic Hamilton-Jacobi equations, Arch. Rational Mech. Anal. 151 (2000) $277-309$.

[36] J. Serrin, The problem of Dirichlet of quasilinear elliptic differential equations with many independent variables, Philos. Trans. Roy. Soc. London Ser. A 264 (1969) 413-469.

[37] P.E. Souganidis, Front propagation: Theory and applications, in: CIME Course on "Viscosity Solutions and their Applications", in: Lecture Notes in Math., vol. 1660, Springer, 1997. 
[38] P.E. Souganidis, Recent developments in the theory of front propagation and its applications, in: G. Sabiclussi (Ed.), Modern Methods in Scientific Computing and Applications, in: NATO Science Ser. II, vol. 75, Kluwer Academic, 2002.

[39] P.E. Souganidis, Stochastic homogenization of Hamilton-Jacobi equations and some applications, Asymptotic Anal. 20 (1999) 1-11. 